\title{
tic\&société
}

Vol. 10, $N^{\circ} 1 \mid 1$ er semestre 2016

Contrôle social, surveillance et dispositifs numériques

\section{Le panoptisme horizontal ou le panoptique inversé}

\section{Simon BOREL}

\section{(2) OpenEdition}

\section{Journals}

Electronic version

URL: http://journals.openedition.org/ticetsociete/2029

DOI: 10.4000/ticetsociete.2029

\section{Publisher}

Association ARTIC

Electronic reference

Simon BOREL, «Le panoptisme horizontal ou le panoptique inversé », tic\&société [Online], Vol. 10, N

1 | 1er semestre 2016, Online since 15 October 2016, connection on 19 April 2019. URL : http://

journals.openedition.org/ticetsociete/2029 ; DOI : 10.4000/ticetsociete.2029

This text was automatically generated on 19 April 2019.

Licence Creative Commons 


\title{
Le panoptisme horizontal ou le panoptique inversé
}

\author{
Simon BOREL
}

\section{Introduction}

1 Quelle est la nature du pouvoir dans la « société en réseaux » (Castells, 2001) ? Cette vaste question amène à explorer les formes complexes que revêtent le contrôle et la surveillance dans un monde où les relations à distance et dans l'immédiateté communicationnelle entre individus connectés l'emportent sur les relations interpersonnelles en face-à-face et sur les relations impersonnelles et fonctionnelles des appareils (État, entreprises marchandes, mass medias). Par surveillance, il faut ici comprendre l'attention concentrée/ciblée, systématique et routinisée de l'attention et de l'observation des habitudes, des conduites de vie, des comportements et des représentations des individus à des fins de domination, d'influence, de pouvoir, de management et/ou de protection (Lyon, 2007).

Deux précisions sont nécessaires avant d'explorer cette question. Sur la définition du pouvoir d'abord. Il s'agit d'éviter plusieurs écueils. Le pouvoir et le contrôle social ne sont pas selon nous réductibles à la vision utilitariste d'une domination d'intérêts (intérêts matériels et/ou symboliques) d'une minorité identifiable - ici, les États ou les géants du capitalisme cognitif - sur une majorité exploitée - les internautes lambda. Il n'est pas non plus pertinent de penser le pouvoir, dans le sillage des surveillance studies (Lyon et Gandy 1993), comme une gouvernementalité évanescente mais omniprésente et omnisciente, dépourvue de centres, de sujets, d'intentionnalités, à l'image du « biopouvoir » foucaldien fondé sur le panoptique et la surveillance disciplinaire intériorisée (Foucault, 1993), ou des «sociétés de contrôle» deleuziennes, "post-panoptiques» et «liquides » (Lyon et Bauman, 2013) basées sur « le contrôle continu » et miniaturisé (Deleuze, 2003). Dès lors, une définition intermédiaire s'accorde pour définir le pouvoir comme l'ensemble des dispositifs opératoires et opérationnels qui se rapportent au politique, au sens de l'autoinstitution de la société. Le pouvoir 
« constitue moins un simple état de fait que le milieu et le moyen à travers lequel se construisent les individus singuliers et collectifs, les classements et les hiérarchies qui les relient les uns aux autres, ainsi que les effets de domination qui apparaissent au sein de ces classements et de ces hiérarchies » (Michon, 2009, pp. 103-104).

Dans ce cadre, des acteurs multiples instituent des modes de relations, de reconnaissance, de conduites via des appareils, des normes, des procédures, des injonctions, des dispositifs et des représentations de l'homme et de la société. Ces derniers émanent prioritairement des élites (économiques, politiques, culturelles), mais nécessitent aussi la participation des individus ordinaires sans le consentement et la participation desquels aucun ordre n'est possible et ne tient.

4 Second préalable : lever les confusions existantes autour de l'avènement concomitant des réseaux/médias sociaux, de la gouvernementalité néolibérale et de la nouvelle dynamique démocratique centrée autour de la reconnaissance (Caillé, 2007). Les réseaux ne sont pas intrinsèquement liés aux nouvelles formes du capitalisme cognitif et à l'« entreprise de soi» (Dardot et Laval, 2009) néolibérale ${ }^{1}$. Mais la dynamique démocratique démocratisation des aspirations à la reconnaissance des singularités, des aptitudes, des compétences, des créativités via la quête de visibilité (Heinich, 2012) - à l'œuvre dans les mondes numériques n'est pas pour autant indépendante des dynamiques néolibérales et "parcellitaires $»^{2}$ contemporaines. Les formes de surveillance contemporaines vont en effet dans le sens d'un contrôle des flux (via, par exemple, l'ensemble des outils du self tracking ou des outils de contrôle de l'activité à distance des salariés) pour promouvoir la compétition (Gane, 2012), l'autoentreprise de soi et l'intériorisation des objectifs mouvants et flexibles du capital.

Penser le contrôle et la surveillance numériques demande d'interroger comment le modèle néolibéral, parcellitaire et marchand d'un côté et les pathologies de la dynamique démocratique de l'autre percutent la société en réseaux, en venant instaurer le panoptisme horizontal.

On gagne à comprendre ce dernier en distinguant trois dimensions qui forment la trame de cet article, la surveillance de tous par tous en ligne, le potentiel renouveau procédural et bureaucratique via Internet, et les nouvelles formes de contrôle marchand des flux désirants sur les réseaux.

\section{La surveillance de tous par tous : être visible par tous, toujours et tout le temps}

7 Le pouvoir en réseaux n'est pas un point fixe omniscient qui surveille et punit comme dans la prison panoptique de Bentham. Il est constitué par des myriades de miroirs qui réfléchissent l'image et l'identité de chacun accessible à tous dans une logique d'individuation individualiste à laquelle concoure la logique néolibérale et "parcellitariste» actuelle. En d'autres termes, nous assistons à l'avènement d'un panoptisme horizontal où tout le monde se surveille, se contrôle, se juge et se jauge sans surveillant général. Certains penseurs parlent de "panoptisme participatif» ou d'« omnioptique » (Kandias, Mitrou et Gritzalis, 2014), d'autres de "canoptique» (Ganascia, 2009) pour désigner ce modèle de « sousveillance » (Mann, Nolan et Wellman, 2003) dans lequel le plus grand nombre observe et voit le plus grand nombre sans aucune autorité centrale. Cette connexion et cette visibilité par tous, toujours et tout le temps, 
sont le propre des liaisons numériques marquées par les quêtes d'authenticité et de transparence contre toute forme de médiation.

\section{Injonction à la connexion et à la confession permanente de soi}

8 La quête de visibilité est la motivation primordiale de l'« individu réticulaire » (Michon, 2009). Les médias sociaux autorisent la constitution par chacun de son propre réseau social dans lequel on peut développer et gérer en "clair-obscur» (Cardon, 2008) son identité, ses créations/talents, ses compétences, ses capacités, l'ampleur de ses relations, ce que l'on accepte de confesser, de montrer, dans un jeu d'inter-reconnaissance permanent en ligne.

Sans disconvenir des dimensions positives de ces dynamiques pour les individus lorsqu'elles s'articulent positivement avec les relations en face-à-face et avec les institutions traditionnelles, la connexité et la visibilité peuvent également conduire à l'affaiblissement de l'intériorité des sujets ainsi que des rythmes langagiers, corporels et sociaux au profit d'une immersion constante, quasiment sans répit, dans les flux informatifs et communicationnels. Être en prise directe avec ses réseaux et être visible par tous, partout, toujours et tout le temps, telle semble être la pente que peuvent emprunter certains individus contemporains les plus insérés dans la sociation réticulaire, et notamment les jeunes générations accoutumées dès l'adolescence aux technologies de la mobilité.

10 Dès lors, on constate une injonction de plus en plus forte à la connexion :

«L'injonction de la mise en réseaux de l'univers social de l'utilisateur fait désormais écho à la peur d'être déconnecté, mis à l'écart de la sphère communicationnelle et sociétale actuelle» (Casilli, 2010, p. 55).

11 De nombreux sondages et témoignages renvoient à cette force des liaisons numériques, en particulier chez ceux qu'on appelle désormais "génération Y ( (Dagnaud, 2011) ou digital natives ${ }^{3}$, en permanence en train d'écrire, d'envoyer et de recevoir des SMS dans un flux conversationnel continu du matin au soir et/ou d'entretenir rituellement sa page Facebook, de consulter les messages, commentaires et photos déposés par leurs « amis ».

La connexion s'accompagne aussi fréquemment de la confession de soi, sans anonymat, sur les réseaux sociaux. Pour G. Lovink, théoricien des médias, « les gens croient que leur libération exige d'eux qu'ils "disent la vérité ", qu'ils se confessent à quelqu'un, un prêtre, un psychanalyste ou un blog et le fait de dire cette vérité va les libérer » (Lechner, 2008).

13 Par conséquent, « disparaitre est devenu impossible, et les intrications entre notre vie physique et notre vie virtuelle sont devenues si ténues qu'il semble impossible aujourd'hui de les séparer complètement» (Piotet, 2011, p. 95). Ces dynamiques redéfinissent ainsi les contours du débat autour de la « société de surveillance».

\section{Aliénation volontaire et tyrannies de la visibilité}

14 Il n'est en effet plus possible de concevoir la surveillance agissant du haut vers le bas par l'intermédiaire d'institutions disciplinaires. Celle-ci s'horizontalise et se généralise audelà des institutions affaiblies. On est confronté à ce que Fabien Granjon appelle une 
société de "participatory surveillance, de peer-to-peer monitoring ou d'interpersonal watching "

« où l'individu n'est plus nécessairement et seulement un sujet surveillé et inquiet de cette surveillance, mais peut aussi tenir le rôle d'un surveilleur curieux (intrusif) [...], de ses pairs ${ }^{4}$, voire de sa propre personne » (2011, p. 78).

Et il est d'autant plus soumis à une surveillance qu'il expose beaucoup de soi en ligne. Ces " conduites volontaires visant à être vu, écouté, répertorié, cité et si possible adulé " (Jauréguiberry, 2011, p. 132) relèvent dès lors non pas de la « contrainte » mais, dans les pas de La Boétie (1995), de la "servitude volontaire à s'exposer». Internet "permet le décentrement de la surveillance par dissémination des points d'observation, ce qui transforme la société en un espace ouvert à l'observation permanente et mutuelle » (Laval, 2012, p. 65).

16 En effet, ce panoptisme horizontal sur les réseaux sociaux est d'autant plus puissant qu'il est lié non pas à une volonté de punir et de redresser les torts de l'individu mauvais citoyen et calculateur se sentant épié par l'œil fixe du pouvoir, mais à la quête de visibilité/célébrité. Il y a un renforcement de l'accent mis sur le contrôle plutôt que sur la discipline. L'analyse prédictive - prévoyance et anticipation - prime sur l'impératif de surveiller et punir (Lyon, 2014). Ainsi, «nous sommes nombreux à prendre part à ce nouveau genre de contrôle sur une base volontaire, souvent sans être conscients de son ampleur » (Christensen, 2010, p. 53).

Cette surveillance tous-tous passe ainsi par la nécessité de rendre des comptes sur ce que nous faisons et où nous nous trouvons. Ou bien encore par la confession d'une partie importante de nos expériences privées sur notre profil Facebook, qui peuvent être scrutées par des (plus ou moins) proches ${ }^{5}$ et qui peuvent tourner en notre désavantage en cas de conflits interpersonnels. Des parties de nous nous échappent et peu importe si nous y faisions bonne, mauvaise ou piètre figure.

Dès lors, si la surveillance de tous par tous peut se comprendre comme la dynamique démocratique poussée à son paroxysme, elle peut s'apparenter également à de nouvelles «tyrannies de la visibilité » (Aubert et Haroche, 2011) poussant au conformisme sous prétexte d'hétérogénéité généralisée. À force de trop vouloir être soi-même indépendamment des masques sociaux et des médiations symboliques, l'individuation réticulaire peut aboutir paradoxalement à imposer des normes de conduites validées par ses pairs en ligne. Or, puisqu'il y a de moins en moins d'écart entre la face sociale et le « moi », la mise à distance vis-à-vis de son propre rôle s'avère plus difficile, et le souci de garder la face un enjeu de tous les instants, poussant parfois à des formes intenses de conformisme. Un « contrôle social » s'exerce à l'encontre des profils proposés.

19 Et si les réseaux peuvent constituer une possibilité de reconnaissance accrue des «facettes» de soi, ils peuvent tout aussi bien se retourner contre soi dans une " autoréification », où les " affects », les « envies », les « buts » apparaissent " comme des objets malléables que l'on peut mobiliser à des fins utilitaires» (Granjon, 2011, p. 81). Pour S. Tisseron, on serait ainsi "passé en quelques dizaines d'années d'une société centrée sur le refoulement »- la "culpabilité » du sujet névrosé moderne - à «une société centrée sur le clivage» (Belhomme, 2010, p. 38) - la "honte» de l'individu réticulaire exposé et visible. La "nécessité de se rendre visible», la «médiatisation permanente », l'injonction à « une production continue et illimitée de soi » qu'impliquent les normes sociales actuelles couplées aux médias et aux technologies numériques sont 
autant de conditions indispensables pour exister socialement. Elles sont aussi bien vectrices d'émancipation (décentrement des appartenances et des identités oppressives) que d'aliénation de la personne (désubjectivation et désymbolisation des individus liquides).

D'autant plus que l'individu réticulaire est parfois son propre despote quand il livre, sur une base quasi volontaire, des myriades de données personnelles dont les grandes firmes du numérique et les États se servent.

\section{Le renouveau procédural et bureaucratique à l'âge digital}

Nous assistons en effet à des collusions fréquentes entre l'individualisation réticulaire, le contrôle marchand des rythmes et des flux désirants, et l'impersonnalité procédurale des réseaux. Celles-ci conduisent à des effets contradictoires: entre émancipation individuelle (mouvement, liberté, ubiquité, désenfermement) et aliénation (obligation de résultats, disponibilité permanente, devoir d'engagement, exigence de réactivité) dans une véritable «sophistication » et « extension » des «mécanismes disciplinaires » (Isaac et Leclercq, 2013).

\section{Les nouveaux dispositifs réticulaires bureaucratiques}

Nos sociétés connaissent l'avènement d'un registre procédural et bureaucratique d'un genre nouveau, moins marqué par l'adhésion à une organisation dans laquelle l'individu est réduit à être un vulgaire rouage interchangeable que par l'inscription dans des dispositifs et des contrôles de flux (Munro, 2000) visant à gérer le détachement des individus réticulaires ${ }^{6}$.

23 Les technologies de la mobilité et les médias sociaux numériques donnent corps à l'aspiration à l'empowerment des usagers, administrés, travailleurs et/ou consommateurs : aptitude et capacité des individus et/ou des groupes à choisir (Alsop et Heinsohn, 2005) et à obtenir/voir les résultats de leurs actions ; sentiment d'accroissement de l'habileté et des compétences; capacité de réaliser un arbitrage entre le souhaitable et le possible dans la lignée de la logique des capabilities (Sen, 1993).

Mais cette recherche de liberté individuelle et d'hétérogénéité à tout prix implique des modes d'agencements flexibles (des travailleurs, des citoyens, des contribuables, des usagers etc.), qui sont mis en œuvre par les organisations rationnelles qui se multiplient plutôt qu'elles ne disparaissent (États, multinationales, organisations de la gouvernance internationale en réseaux). On voit en effet se multiplier les modèles de néomanagement, de gestion des ressources humaines, de participation à l'élaboration de projets, qui, sous prétexte d'empowerment, déploient un arsenal de contrôles, d'objectifs, d'évaluation comptable, de procédures administratives, utilisant les techniques réticulaires (informatiques en ligne et surveillance électronique) comme support, et qui conduisent les individus à se représenter et à agir comme étant des points du réseau, responsables, évaluables, objectivables et connectables avec tous les autres.

Ainsi,

" les réseaux donnent un moyen de contrôle puissant sur les internautes ou les citoyens et les consommateurs [permettant] de réunir tellement d'informations sur un 
internaute » que des éléments de la vie privée ${ }^{7}$ « sont disponibles très largement au toutvenant ou deviennent objet d'échanges commerciaux et de pub»(Padis, 2011, p. 80) ${ }^{8}$.

De cette manière, les individus réticulaires, bien loin d'une pleine et entière maîtrise de leur autonomie, sont pris dans les filets d'un nombre croissant de réseaux de service ou d'évaluation croisés dont ils maîtrisent de moins en moins les tenants et les aboutissants sociotechniques.

\section{Les nouveaux dispositifs numériques privé et public}

Ces nouveaux dispositifs numériques ont un impact contradictoire sur le travail. Ils permettent d'un côté l'émancipation relative de l'activité de travail des modèles panoptiques industriels classiques via les technologies de la mobilité numérique (permettant "d'être connectés au système d'information de l'entreprise » et d'être joignable à tout moment où que l'on se trouve), qui autorisent à la fois des " espaces d'autonomie » dans le travail et des «formes de contrôle » (Le Douarin, 2007) du travail par les travailleurs eux-mêmes. Mais ils rendent possible d'un autre côté :

« un contrôle de l'activité des salariés dans un contexte de mouvement et de délocalisation (travail hors de l'enceinte de l'entreprise). La surveillance est tout aussi permanente dans ses effets, mais elle s'adapte aux contours de l'entreprise hypermoderne. Elle suit les salariés dans ses moindres mouvements, à l'intérieur comme à l'extérieur de l'entreprise » (Noël, 2007, p. 59).

Les TIC peuvent ainsi permettre au management des organisations «de continuer le processus de rationalisation et d'organisation scientifique du travail » dans « l'activité de traitement de l'information, et, tout particulièrement, l'activité de coordination, c'est-àdire les relations de travail, donc le social» (Craipeau, 2009, p. 177). La "gestion des connaissances, supportée par un système électronique » devient «le fondement d'un mode d'organisation qui cherche à remplacer les anciennes formes de collectifs par de nouvelles formes de type réseau, que l'on habille d'ailleurs du mot de communauté » (ibid. ). Ces nouvelles formes, comme le groupware ou «télétravail» renforcent «l'individualisation, voire l'atomisation du travail», favorisent la «coordination» (dimension fonctionnelle) plutôt que la «solidarité » (dimension sociosymbolique) et gomment les « médiations».

"Grâce aux réseaux de télécommunication, l'entreprise se simplifie jusqu'à devenir un assemblage temporaire d'individus caractérisés par les deux qualités propres à la nouvelle entreprise en réseaux : la mobilité et l'engagement professionnel de type entrepreneurial » (ibid., p. 180).

Ces contrôles et opérations de rationalisation réticulaires sont également l'œuvre des États en direction des multiples flux de population. Par exemple, les politiques migratoires européennes se sont adaptées, constatant la faillite des «méthodes traditionnelles de gestion des flux». Elles ont eu alors "recours aux technologies nouvelles d'identification et de surveillance» qui sont «à la fois mobiles et intelligentes ", " capables de s'adapter à la mobilité des individus, de les suivre, de tracer leur itinéraire et de déterminer leur véritable identité »(Ceyhan, 2013, p. 134). On peut alors parler de «frontières intelligentes " (flexibles et mobiles) 9 pour qualifier ces nouveaux dispositifs étatiques visant à « adapter la sécurité à la fluidité » via " un réseau de surveillance technologique », des «technologies biométriques d'identification » et des 
bases de données interconnectées ${ }^{10}$, visant notamment à établir « un régime de triage de migrants en fonction de leurs intentions ».

Plus largement, les réseaux sociaux, bien loin de revêtir systématiquement les traits de l'émancipation démocratique, peuvent s'avérer être de précieuses armes de surveillance et de répression au service des États - dans les pays dictatoriaux mais aussi démocratiques.

\section{Renouveau et affinage de la surveillance étatique}

Le politiste E. Morozov (2011) combat l'idée selon laquelle la Toile aurait la capacité de «libérer les peuples opprimés» et de les faire accéder à la démocratie. Pour lui, "Internet peut être un instrument de libération comme il peut être un instrument d'oppression » (Morozov in Millot, 2011).

Instrument de libération? Le « synoptique " (Mathiesen, 1997) à l'œuvre dans les médias sociaux numériques permet en effet au plus grand nombre de garder la minorité sous observation. Ceci est particulièrement vrai dans les pays aux régimes autoritaires dont la contestation se répand, non sans de nombreuses et violentes contradictions, dans le monde (notamment récemment en Tunisie et en Égypte, puis en Ukraine). Ces logiques contestataires conduisent de nombreux peuples et groupes opprimés à investir - malgré toutes les limites propres à la surveillance étatique et au blocage partiel du Web - les outils de sousveillance numérique comme espace virtuel alternatif pour faire vivre les libertés individuelles, publiques et collectives. Ils sont des médiums qui peuvent donner la possibilité à de nombreux individus de faire front à la toute-puissance du pouvoir politique et/ou religieux qui s'oppose à la constitution et à l'existence d'une société civile organisée et revendicative. Ils rassemblent des aspirants citoyens mobilisés ou mobilisant des réseaux de soutien, d'entraide et de paroles contre la censure et la répression (Granjon, 2011).

33 En Occident, on assiste plutôt au fleurissement de micropouvoirs indépendants dans une logique de "citoyenneté de défiance » et de "contre-démocratie » (Rosanvallon, 2006), dans lesquelles le citoyen exerce un pouvoir de surveillance du pouvoir politique. Les individus "s'organisent autour des trois actions de vigilance, de dénonciation ${ }^{11}$ et de notation" (ibid., p. 68). La "démocratie coopérative" met en œuvre des expérimentations qui s'organisent "autour d'un dispositif permettant d'agir et de coopérer » (via notamment la libération des « données publiques») de manière souple, transparente et autonome, et organisant des « zones d'expertise ou de discussion à côté des institutions, voire contre elles» (Cardon, 2010, p. 84). Ce dispositif autorégulé est souvent représenté par des "procédures de surveillance et de sanction", une "surveillance participative» décentralisée apte à réguler les comportements et la conflictualité en ligne.

Cependant, si la " Toile a permis aux jeunes de partager leurs griefs, les a galvanisés », les "régimes autoritaires ont aussi su tirer les leçons de ces soulèvements" (Morozov, interrogé dans Libération, 5 mars 2011). Le régime iranien par exemple

« a pris de nombreuses mesures pour mieux contrôler les nouveaux médias. Plusieurs unités de police s'y consacrent, le gouvernement a son propre réseau social, le régime forme des blogueurs conservateurs ou religieux, et peut aussi mener des attaques cybernétiques » (ibid.). 
« Au Soudan, les services secrets ont lancé sur Internet des appels à de fausses manifestations, juste pour voir qui descendrait dans la rue et arrêter les protestataires. » ${ }^{12}$ De son côté, « le pouvoir chinois déploie des moyens colossaux pour contrôler Internet et les réseaux sociaux. Le sociologue Gary King, d'Harvard, qui a dirigé une excellente étude sur le sujet, y voit l'“effort le plus important jamais mis en œuvre pour censurer sélectivement l'expression humaine“" (Kauffmann, 2009).

«Et il les contrôle d'une manière extrêmement sophistiquée, en laissant les réseaux sociaux jouer leur rôle de soupape, lorsque les tensions sont trop vives. Internet est aussi un formidable outil d'information pour le pouvoir, qui peut prendre en permanence la température de la population » (ibid.).

Mais les gouvernements ne sont pas seuls à l'œuvre. Ils peuvent compter sur leur propre population ${ }^{13}$ ou « déléguer la censure à des firmes locales. Ainsi, les autorités chinoises demandent aux compagnies qui hébergent des sites de censurer les contenus antigouvernementaux » (Morozov in Millot, 2011). L'État chinois peut également compter sur la coopération technologique de certains États occidentaux ${ }^{14}$, prompts à donner des leçons de démocratie: "Les États-Unis accusent les Chinois d'entraver la liberté d'expression et leur vendent les technologies comme Narus qui permettent de filtrer la Toile » (ibid.).

Mais les États-Unis ne s'arrêtent pas là. Outre l'utilisation des données à des fins sécuritaires - telle l'utilisation de "logiciels de sécurisation prédictives $»^{15}$ dans de nombreuses villes américaines - ils sont également à l'œuvre dans l'élaboration d'une surveillance planétaire des télécommunications des particuliers et de certains États via les grandes entreprises multinationales du numérique qui relativise la thèse de l'émancipation par la sousveillance citoyenne. En effet, le programme de surveillance "Prism », révélé au grand public dans le Guardian, le Washington Post et le New York Times en juin 2013, sur la base des révélations du lanceur d'alerte et ancien collaborateur de la CIA E. Snowden, " permet à la NSA (National Security Agency) de se connecter aux serveurs des entreprises ${ }^{16}$, via un portail, pour consulter des informations sur des utilisateurs dont il existerait des éléments permettant de penser "raisonnablement" qu'ils sont à l'étranger, le tout sans ordonnance de justice » (Le Monde, AFR, Reuters, 2013).

«Le but avoué de ce programme mis en place par le gouvernement américain depuis 2007 est de lutter contre les activités et la menaces terroristes, les cyberactivités hostiles ou encore la prolifération nucléaire, mais pourrait s'étendre potentiellement à de nombreux autres motifs plus inavouables. Comme les entreprises privées peuvent utiliser les données privées à des fins commerciales, les États, en lien avec ces entreprises, peuvent les utiliser à des fins politiques. D'autant plus que la collecte d'informations auprès de ces grandes entreprises concerne « les courriels, les fichiers hébergés (par exemple sur Google Drive), les photos, le contenu des communications (texte, audio, vidéo) ou encore des actions précises, comme la connexion au compte utilisateur " (Le Monde, 2013).

Autant de données dont la compilation permet d'obtenir une cartographie précise des relations, des convictions et des pratiques individuelles. Cartographie qui échappe dans une grande mesure aux particuliers pris dans ce «nonoptique » (Vaidhyanathan, 2008) dans lequel on ne sait jamais que l'on est surveillé, par qui, avec quelles intentions et quel degré d'indiscrétion ${ }^{17}$. 

institutions traditionnelles, prenant appui sur la surveillance horizontale via la "dataveillance ${ }^{18}$ » (Degli Esposti, 2014). Certains chercheurs en appellent d'ailleurs à penser la «dialectique entre la discipline foucaldienne et le contrôle deleuzien considérant une possible hybridation de ces dispositifs de contrôle » (Isaac et Leclercq, 2013).

Même s'il apparait inopportun (bien que tentant) d'assimiler cette affaire au Big Brother totalitaire orwellien - les contre-pouvoirs démocratiques et les libertés individuelles et publiques sont de fait à l'œuvre dans la révélation de cette affaire -, elle révèle néanmoins les dérives potentielles du pouvoir de contrôle réticulaire des entreprises et des États dans le monadisme parcellitaire contemporain. Si « Prism » n'est pas assimilable à un programme disciplinaire, voire totalitaire de type benthamien, il est cependant, comme lui, soucieux d'accéder et de garantir le «plus grand bonheur du plus grand nombre ».

40 Si la rationalité procédurale peut gagner du terrain, c'est le cas également pour la rationalité marchande et consumériste.

\section{Le contrôle marchand des flux désirants}

41 Sur les décombres de l'économie et de la société industrielle standardisée, fonctionnelle, hiérarchisée et verticale se font jour une économie et une société informationnelle plus contributive, participative, flexible et horizontale, qui peut conduire à des formes d'émancipation individuelle et d'autonomisation, mais aussi à de nouvelles formes de contrôle et d'aliénation.

\section{Triomphe de l'économie participative}

(Béraud et Cormerais, 2011) et collaboratives (Botsman et Rogers, 2011) ont le vent en poupe depuis et avec l'émergence du Web 2.0 et $\mathrm{du}$ peer-to-peer. Ces pratiques socioéconomiques se caractérisent par l'émergence de formes de production, d'échange, de financement et de consommation reposant sur la mise en relation directe, intermédiée par une plate-forme numérique (gratuite ou payante), de producteurs, d'usagers et/ou de consommateurs sur la base de l'échange marchand (achat et revente), du don, du troc, de la mutualisation, du prêt, sans passer par les intermédiaires marchands et industriels classiques.

Pour certains, l'« économie de la contribution », (Béraud et Cormerais, 2011), qui recouvre «un ensemble de pratiques spécifiques», renvoie «aux participations de contributeurs librement investis dans l'activité et qui acceptent de coopérer et de diffuser leurs connaissances sans attendre de contrepartie sous la forme d'un équivalent monétaire » (ibid., p. 164), échappant ainsi aux disciplines marchandes classiques. En tant qu'« économie générale » à l'ère du numérique, l'économie de la contribution est une « régulation par la participation », appuyée sur la «compétence des contributeurs » et caractérisée par « le degré d'interaction des participants à l'intérieur d'une activité » (ibid ., p. 167). La vulgarisation de connaissances par les contributeurs repose sur un modèle de partage et de diffusion d'informations collaboratif et égalitaire selon lequel «tout participant détient une parcelle de compétence » (Flichy, 2010) du moment qu'il est doté 
d'une « capacité argumentative ». Dès lors, les clients et les consommateurs deviennent de plus en plus des « prosommateurs » qui s'émancipent des produits et des modes de vie que le marché conçoit pour lui.

Cependant, ces nouveaux modèles socioéconomiques peuvent aussi faire l'objet de nouvelles formes de contrôle marchand, pénétrant en profondeur les subjectivités en ligne sur la base d'une aliénation (quasi) volontaire.

\section{Exploitation protocolaire et attentionnelle}

45 Certains penseurs parlent d'« exploitation protocolaire » (Wark, 2013) pour qualifier le système dans lequel la «classe vectorialiste " $^{19}$ détient « le contrôle de la logistique » et contrôle la manière dont les supports, les flux, l'accès, le stockage d'informations et de données sont gérés. Elle a ainsi le " pouvoir de calculer », de " déplacer l'information d'un endroit à un autre» et "de réaliser la valeur de ce que nous produisons » (ibid.), en coordonnant notamment «tous les aspects de la vie sous l'emprise de la marque, du brevet et du droit d'auteur" (ibid., p. 193). Les détenteurs du pouvoir à l'ère de l'information sont les " commutateurs", qui contrôlent les "points de connexion entre différents réseaux stratégiques ", et les " programmateurs » ou les « réseaux d'acteurs qui détiennent le pouvoir de créer des réseaux » (Castells, 2013).

Autre forme de dispositif employé par le «capitalisme mental» (Francq, 2013), l'« exploitation attentionnelle» met en œuvre une économie médiatique de la visibilité. Le capitalisme mental privatise l'«espace d'expérience» (public et commun) via l'émergence des marchés de l'« attention » et de la "considération ». Ainsi, les médias «capitalisent la considération» et l'« information disponible» qu'ils diffusent et se paient en « attention » dont veulent profiter les annonceurs publicitaires, « le service de l'attraction » s'échangeant contre de l'argent.

Dès lors, les dominants de la nouvelle économie de la visibilité sont «ceux dont les revenus en attention excèdent de plusieurs ordres de grandeur leurs dépenses en attention » (ibid., p. 203). Et le «type d'exploitation qui caractérise le capitalisme mental s'exerce à l'encontre de ceux, en très grand nombre, qui prêtent toujours attention et considération, mais qui n'en reçoivent guère en retour » (ibid., p. 212). Se déchaîne par conséquent une "lutte générale pour la considération » qui «mène à la production en masse de moyens d'accéder à la visibilité, ainsi qu'au développement de moyens permettant d'enregistrer le revenu d'attention " (ibid.) dans un contexte où les luttes pour la démocratisation de la reconnaissance sont aussi une dynamique essentielle des réseaux.

Outre ces nouveaux dispositifs de contrôle du capitalisme cognitif mondialisé, le Web peut également être une bénédiction pour étendre les champs de la consommation, l'influence et l'image de marque des firmes.

\section{Contrôle marchand des données personnelles}

L'« offre via le Net » de " produits culturels et informatifs » « est devenue quasi illimitée » et fait glisser le consommateur avec son consentement et parfois son enthousiasme vers le "vertige de l'hyperchoix » (Dagnaud, 2010) : «toujours plus d'aventures, de plaisirs inédits », « d'information sur vous et vos hobbies ». Les technologies numériques alliées à 
la société de marché consumériste "plongent l'individu dans la démesure », en lui donnant :

«l'illusion de la toute-puissance : tout voir, tout savoir, accéder à toutes les

connaissances, immédiatement, accumuler les biens immatériels (musiques, textes, vidéos, informations etc.), multiplier les contacts, aller toujours plus vite, être disponible en permanence » (Biagini, 2012, p. 362).

Les consommateurs sur Internet sont tantôt conviés à participer à l'élaboration de l'offre en donnant leur avis sur leurs exigences/préférences, tout en adhérant à des marques et à des identités de marché, tantôt tracés, pistés, profilés pour mieux être cernés en tant que cible publicitaire et se voir proposer les services qui leur correspondraient le mieux.

51 Ainsi, de plus en plus d'entreprises «ont recours à des sites Web participatifs pour associer les consommateurs à la conception de leurs produits et services et à leur communication » (Lelong et Gayoso, 2010, p. 99). Il s'agit de :

" convaincre et d'impliquer un certain nombre de consommateurs clés (souvent appelés influencers dans le jargon marketing) en leur fournissant les outils et les supports (par exemple, une série de vidéos virales) qui leur permettront de communiquer à leur réseau l'enthousiasme qu'ils auront pour un produit » (Loveluck, 2008, p. 164).

Des conseils de solutions aux entreprises insistent sur l'importance "de mesurer le nombre d'expositions - donc l'influence qui peut s'en dégager» - de ses fans à une campagne » sur Facebook, mais également - et surtout - sur les «amis» de ses fans : " amener un fan à “aimer"votre marque ${ }^{20}$ n'est pas une fin en soi, mais le début de votre relation sociale avec ce consommateur" (Gatignol, 2011). Le consommateur doit donc « devenir un militant de la marque, un co-créateur enthousiaste de son succès » et former « des communautés de marques » (Biagini, 2012, p. 305).

Ainsi, les qualités relationnelles d'Internet se trouvent détournées à des fins marchandes :

« les flux d'informations et de données doivent circuler de plus en plus librement à

l'intérieur de l'entreprise, comme entre l'intérieur et l'extérieur, si l'entreprise tient à intégrer le mode collaboratif et relationnel dont elle a besoin pour rester compétitive » (Pisani et Piotet, 2008, p. 197).

De leur côté, les «fournisseurs de service » et les "plates-formes d'intermédiation sur l'Internet» pratiquent en toute liberté le profilage et la monétisation des données personnelles. Ainsi, par exemple, Facebook:

« monnaye les données que l'internaute indique sur sa page à travers le réseau de ses amis, dans un processus de mécanique virale, tel un virus, le message publicitaire se propage et se diffuse à toute une série d'internautes interconnectés » (Benhamou, 2011, p. 104).

C'est entre ces reflets infinis que donnent à voir les individus connectés à la plate-forme que s'engouffrent les formes de contrôle et d'appropriation marketing et marchand, Facebook ne cachant pas « son intention de connaître le plus possible d'éléments sur nous pour pouvoir nous profiler et nous proposer une publicité plus susceptible de nous intéresser » (Piotet, 2011, p. 91). Grâce à la géolocalisation, les annonceurs peuvent également connaître les lieux que fréquentent les consommateurs, «à quel rythme, leur manière de se déplacer dans la ville et même qui ils rencontrent, quand, où, pendant combien de temps » (Biagini, 2012, p. 304). 

Google, vendent aux annonceurs les mots-clés de recherche des internautes dans un gigantesque marché du référencement vecteur de quantification généralisée. Les « fermes de contenu» qui se multiplient sur Internet permettent ainsi d'«identifier ce que le public veut lire » d'après la fréquence des termes tapés dans les moteurs de recherche, qui sont aussi les «mots-clés les plus demandés par les publicitaires », puis d'« enrôler des masses de rédacteurs pour le produire ", instituant par là même le principe d'une «information low cost» favorisant les moteurs de recherche, des grands «sites de contenus low cost » qui « recherchent surtout de gros volumes d'audience pour vendre de la publicité à très faible prix et miser sur une "économie du clic" " (Ramonet, 2011, p. 27).

\section{Essor de l'autocontrôle}

Outre le contrôle marchand des flux désirants, on assiste également, dans le sillage de l'Internet des objets connectés (dans des secteurs aussi divers que l'énergie, la santé, le sport, etc.), à la montée en puissance d'une logique d'autocontrôle, de maîtrise, d'évaluation et de quantification de soi et du soi. Celle-ci peut s'interpréter aussi bien à l'aune des quêtes identitaires et de l'individu psychologique hypercontemporain, qui cherche de la reconnaissance de sa singularité par la mise en visibilité et l'exposition de soi, que de la gouvernementalité néolibérale et du "solutionnisme technologique» (Morozov, 2013).

La gouvernementalité par les algorithmes est basée à la fois sur l'autocritique et le contrôle du self. Les technologies de self tracking (capteurs d'activité, enregistreurs de sommeil, balances portables, applications mobiles, etc.), notamment, permettent l'enregistrement, la mesure, la visualisation et la publication de données qui sont autant d'éléments inséparables d'une relation autorapportée et publicisée à soi, semblable à la confession et à l'aveu. Dès lors, la discursivité numérique s'articule autour de la mesure comme objectivation de soi, du dépistage réticulaire comme relation à soi et de la visualisation des traces numériques comme explication et découverte de sa propre intériorité (Reigeluth, 2014). L'essor des techniques de « quantification » ou de « métrique de soi $^{21} »$ se présente en effet :

« comme l'un des instruments mis à la disposition du sujet contemporain pour motiver une action autonome, poser des objectifs personnels et se donner les moyens de les atteindre, autrement dit comme l'une des incarnations de cette injonction à l'autogestion de soi et l'un des moyens de s'y conformer » (Pharabod et al., 2013, p. 117).

Quantification et calcul de soi qui s'effectuent bien sûr toujours sous le regard (approbatif, réprobateur, admiratif, sceptique, etc.) des autres en ligne :

" soit parce que les traces calculées individuelles sont visibles pour d'autres en situation de produire leurs propres commentaires et évaluations, soit parce que les calculs individuels sont nichés dans des représentations qui figurent d'une manière ou d'une autre les données d'autrui » (Licoppe, 2013, p. 50).

Par conséquent, le système marchand s'approprie le réservoir de travail quasi gratuit et les traces/données personnelles laissées derrière eux par les internautes. Mais ces derniers collaborent, voire adhérent volontairement aux modes de consommation participatifs qui rejoignent les quêtes identitaires et le libéralisme culturel ambiants.

tic\&société, Vol. 10, Nㅜ 1 | 1 er semestre 2016 


\section{Conclusion}

61 Le panoptisme horizontal semble de plus en plus être la forme prise par le pouvoir dans la société en réseaux. Il ne faut cependant pas se méprendre sur ses causes ni sur ses caractéristiques.

Ce panoptisme réticulaire en ligne émerge à l'intersection de plusieurs dynamiques : les médias sociaux numériques, le capitalisme informationnel et les géants du numérique, la gouvernementalité néolibérale et la dynamique démocratique contemporaine. Il apparaît particulièrement difficile de retracer avec certitude leurs liens et leurs agissements réciproques. Tout au plus peut-on affirmer qu'ils avancent conjointement, avec des affinités électives, sans que, pour l'instant, aucun ne prenne définitivement le dessus sur l'autre - ni économisme, ni déterminisme technologique, ni primat de la dynamique sociopolitique et subjective.

Les caractéristiques de ce panoptisme apparaissent en revanche plus clairement : surveillance et contrôle de tous par tous sur les réseaux via la quête de visibilité/célébrité en ligne, renouveau des dispositifs de surveillance étatiques via Internet, et émergence de contrôles marchands des activités sociales médiatisées et des flux désirants consuméristes. Ces caractéristiques ne sont pas assimilables au modèle totalitaire. Bien au contraire. Elles se posent même en rupture avec lui - même si elles mêlent des formes panoptiques anciennes et des outils de surveillance traditionnels. Mais ce symétrique partiellement inversé conserve certaines pathologies fondamentales du totalitarisme, d'autant plus dangereuses qu'elles se réalisent au nom de la liberté individuelle. Ainsi, l'idéal de transparence des rapports sociaux à eux-mêmes contre toute intériorité et socialité, l'obligation communicationnelle permanente à laquelle il s'agit de se conformer au risque, le cas échéant, de disparaître socialement, l'existence d'un monde fictif déterritorialisé et désymbolisé dans lequel les simulacres se conjuguent dans une déréalisation de plus en plus poussée, sont les signes manifestes que l'illimitation individuelle et économique alliée au numérique est synonyme de nouvelles pathologies psychiques et collectives.

ALSOP R. et HEINSOHN N., 2005, « Measuring Empowerment in Practice: Structuring Analysis and Framing Indicators », World Bank Policy Research Working Paper, $123 \mathrm{p}$.

AUBERT N. et HAROCHE C., 2011, Les Tyrannies de la visibilité: Être visible pour exister ?, Toulouse, Érès.

AZAM J., 2013, Facebook. Anatomie d'une chimère, Toulouse, Collectif des métiers de l'édition, coll. « Les Réveilleurs de la nuit ».

BAIN P. et TAYLOR P., 2000, "Entrapped by the "Electronicon Panopticon" ? Resistance in the Call Center, New Technology ", Work and Employment, vol. 15, n 1, pp. 2-18.

BAUMAN, Z. et LYON D., 2013, Liquid Surveillance: A Conversation, Cambridge, Polity.

BELHOMME L., 2010, « Interview de Serge Tisseron. L'identité et les liens transformés par les nouvelles technologies », Cahiers de psychologie clinique, $n^{\circ} 35$, pp. 31-55.

BENHAMOU F., 2011, «L'État et l'Internet : Un cousinage à géométrie variable », Esprit (juillet), n - 376, pp. 96-110.

BÉRAUD P. et CORMERAIS F., 2011, «Économie de la contribution et innovation sociétale », Innovations, $\mathrm{n}^{\circ} 34, \mathrm{pp}$. 163-183. 
BIAGINI C., 2012, L'Emprise du numérique : Comment Internet et les nouvelles technologies ont colonisé nos vies, Montreuil, L'Échappée.

BOTSMAN, R. et ROGERS, R., 2011, What's Mine Is Yours : How Collaborative Consumption is Changing the Way We Live, Londres, HarperCollins.

CAILLÉ A. (dir.), 2007, La Quête de reconnaissance: Nouveau phénomène social total, Paris, La Découverte.

CAILLÉ A., 2006, « Un totalitarisme démocratique ? Non, le parcellitarisme », in A. CAILLE (dir.), Quelle démocratie voulons-nous? Pièces pour un débat, Paris, La Découverte.

CARDON D., 2012, « Montrer/regarder : L'Économie de la visibilité sur les réseaux sociaux d'Internet ", in C. JANSEN et J. MARQUET (dir.), Lien social et Internet dans l'espace privé, LouvainLa-Neuve, Harmattan-Academia, pp. 21-50.

CARDON D., 2010, La Démocratie Internet : Promesses et limites, Paris, Le Seuil-La République des idées.

CARDON D., 2008, « Le Design de la visibilité. Un essai de cartographie du Web 2.0 », Réseaux, n -152, pp. 93-137.

CASILLI A. , 2011, « Trois idées reçues sur Internet », Sciences humaines, n² 229, pp. 27-29.

CASILLI A. 2010, «"Petites boîtes” et individualisme en réseaux : Les usages socialisants du Web en débat, Réalités industrielles, pp. 54-59.

CASTELLS M., 2013, Communication et pouvoir, Paris, Éditions de la Maison des sciences de l'homme.

CASTELLS M., 2001, La Société en réseaux : L'Ère de l'information, Paris, Fayard.

CEYHAN A., 2010, «Les Technologies européennes de contrôle de l'immigration. Vers une gestion électronique des personnes à risque ", Réseaux, n 159, pp. 131-150.

CHAMPAGNE A., 2012, « Surveillance profonde sur Internet », Le Monde diplomatique (janvier), $\mathrm{n}$ -694, p. 23.

CHARDEL P.-A. (dir.), 2014, Politiques sécuritaires et surveillance numérique, Paris, CNRS Éditions, coll. « Les Essentiels d'Hermès ».

CHRISTENSEN M., 2010, « Facebook is watching you », Manière de voir, nº 109, pp. 53-55.

CITTON Y., 2013, «Économie de l'attention et nouvelles exploitations numériques », Multitudes, n ०54, pp. 163-175.

COLL S., 2014, « La Vie privée comme outil de gouvernance : Surveiller et fidéliser le lien marchand ", Les Cahiers du numérique, vol. 10, pp. 45-68.

CRAIPEAU S., 2009, « Le Management cognitif », dans S. CRAIPEAU, G. DUBEY, P. MUSSO et B. PAULRÉ (dir.), La Connaissance dans les sociétés techniciennes : Enjeux et dangers de l'industrialisation de la connaissance, Paris, L'Harmattan, pp. 175-188.

DAGNAUD M., 2011, Génération Y : Les Jeunes et les réseaux sociaux, de la dérision à la subversion, Paris, Presses de Sciences Po, coll. « Nouveaux débats ».

DAGNAUD M., 2010, « Le Web, ce laboratoire du capitalisme sympa », Le Débat, n 160, pp. 161-176.

DARDOT P. et LAVAL C., 2009, La Nouvelle Raison du monde, essai sur la société néolibérale, Paris, La Découverte. 
DEGLI ESPOSTI, S., 2014, « When Big Data Meets Dataveillance: The Hidden Side of Analytics », Surveillance \& Society, vol. 12, n², pp. 209-225, www.surveillance-and-society.org.

DELEUZE G., 2003, Pourparlers (1972-1990), Paris, Les Éditions de Minuit.

FERNANDEZ B., 2011, « Le Temps de l'individuation sociale », Revue du MAUSS, n 38, pp. 339-348.

FLICHY P. et PARASIE S., 2013, « Présentation », Réseaux, n 178-179, pp. 9-19.

FLICHY P., 2010, Le Sacre de l'amateur : Sociologie des passions ordinaires à l'ère numérique, Paris, Le Seuil-La République des idées.

FOUCAULT M., 1993, Surveiller et punir, Paris, Gallimard, coll. « Tel ».

FRANCQ G., 2013, «Capitalisme mental », Multitudes, n 54, pp. 199-213.

GANASCIA J.-G., 2009, Voir et pouvoir : qui nous surveille ?, Paris, Le Pommier.

GANE N., 2012, « The Governmentalities of Neoliberalism: Panopticism, Post-panopticism and Beyond », The Sociological Review, vol. 60, pp. 611-634.

GATIGNOL D., 2011, « Entreprises : vous ne pouvez pas ignorer la force des fans », Le Monde en ligne, solutionsauxentreprises.lemonde.fr/relation-client/entreprises-vous-ne-pouvez-pasignorer-la-force-des-fans_a-33-281.html, dernière consultation le 19 octobre 2011.

GRANJON F., 2011, « De quelques pathologies sociales de l'individualité numérique : Exposition de soi et autoréification sur les sites de réseaux sociaux ", Réseaux, n 16, pp. 75-103.

HASKI P., 2008, Internet et la Chine, Paris, Seuil-Presses de Sciences Po.

HEINICH N., 2012, De la visibilité : Excellence et singularité en régime médiatique, Paris, NRF Gallimard.

ISAAC H. et LECLERCQ A., 2013, « Technologies de l'information, contrôle et panoptique : Pour une approche deleuzienne ", Systèmes d'information et management, vol. 18, $\mathrm{n}^{\circ} 2$.

JAURÉGUIBERRY F., 2011, « L'Exposition de soi sur Internet : Un souci d'être au-delà du paraître ", in N. AUBERT et C. HAROCHE, Les Tyrannies de la visibilité : Etre visible pour exister?, Toulouse, Érès, pp. 131-144.

KANDIAS M., MITROU L. et GRITZALIS D., 2014, « Social Media Profiling : A Panopticon or Omniopticon Tool ? ", in Proceedings of the 6th Biannual Surveillance and Society Conference (24-26 avril), Barcelone.

KAUFFMANN S., 2009, « Weibo versus Shibada, la dynamique chinoise », Le Monde, 18 juin.

LA BOÉTIE É. de, 1995, Discours de la servitude volontaire, Paris, Mille et une nuits.

LE MONDE, 2013, « Bataille d'informations autour de l'outil de surveillance "Prism" », Le Monde en ligne www.lemonde.fr/technologies/article/2013/06/10/bataille-d-informations-autour-de-loutil-de-surveillance-prism_3427534_651865.html, dernière consultation le 29 février 2016.

LE MONDE, AFP et REUTERS, 2013, « Le FBI aurait accès aux serveurs de Google, Facebook, Microsoft, Yahoo ! et d'autres géants d'Internet », Le Monde en ligne, www.lemonde.fr/ ameriques/article/2013/06/07/le-fbi-a-acces-aux-serveurs-des-geants-dinternet_3425810_3222.html, dernière consultation le 29 février 2016.

LAVAL C., 2012, «Surveiller et prévenir : La Nouvelle Société panoptique », Revue du MAUSS, n -40, pp. 47-72.

LE DOUARIN L., 2007, “ “C'est personnel !” : L’Usage des TIC par les cadres dans l'articulation des temps sociaux : vers une évolution de la rationalisation du travail ? ", L'Homme et la société, $\mathrm{n}$ - 163-164, pp. 75-94. 
LECHNER M., 2008, « Web 2.0 : L'anonymat n'est plus qu'une notion nostalgique », Libération, http://www.liberation.fr/week-end/2008/01/12/1-anonymat-n-est-plus-qu-une-notionnostalgique_62540, dernière consultation le 8 octobre 2016.

LELONG B. et GAYOSO E., 2010, «Innovations avec l'usager et plateformes collaboratives : Des modes d'engagement hétérogènes ", Réseaux, n 164, pp. 97-126.

LICOPPE C., 2013, «Formes de la présence et circulations de l'expérience : De Jean-Jacques Rousseau au "Quantified Self" ", Réseaux, n 182, pp. 21-55.

LOVELUCK B., 2008, « Internet, vers la démocratie radicale ? », Le Débat, n 151, pp. 150-166.

LYON D., 2014, «Surveillance, Snowden, and Big Data : Capacities, consequences, critique », Big Data \& Society, pp. 1-13.

LYON D., 2007, Surveillance Studies : An Overview, Cambridge, Polity Press.

MANN, S., NOLAN J. et WELLMAN B., 2003, « Sousveillance: Inventing and Using Wearable Computing Devices », Surveillance \& Society, vol. 1, éd. 3, pp. 331-355.

MARTIN-JUCHAT F., PIERRE J., 2011, «Facebook et les sites de socialisation : Une surveillance librement consentie ", in GALINON-MÉLÉNEC B. (dir.), L'Homme trace : Perspectives anthropologiques des traces contemporaines, Paris, CNRS Éditions, pp. 105-125.

MATHIESEN T., 1997, « The Viewer Society : Michel Foucault's “Panopticon" Revisited », in Theoretical Criminology, vol. 1, n², pp. 215-234.

MAYER-SCHÖNBERGER V. et CUKIER K., 2013, « Mise en données du monde, le déluge numérique », Le Monde diplomatique (juillet), n 712, pp. 1, 20-21.

MICHON P., 2009, Les Rythmes du politique: Démocratie et capitalisme mondialisé, Paris, Les Prairies ordinaires.

MILLOT L., 2011, « Le Net, instrument de libération et d'oppression », Libération en ligne, www.liberation.fr/monde/01012323705-le-net-instrument-de-liberation-et-d-oppression, dernière consultation le 29 février 2016.

MOROZOV E., 2013, To Save Everything, Click Here: Technology, Solutionism, and the Urge to Fix Problems that Don't Exist, Londres, Allen Lane.

MOROZOV E., 2011, The Net Delusion : The Dark Side of Internet Freedom, New York, Public Affairs.

MUNRO I., 2000, « Non-disciplinary Power and the Network Society”, Organization, $\mathrm{n}^{\circ}$ 7, pp. 679-695.

NOËL C., 2007, « La Cybersurveillance au travail, une nouvelle version du panoptisme managerial ? ", Humanisme et Entreprise, $n^{\circ} 285$, pp. 49-64.

PADIS M.-O., 2011, « Enjeux techniques, modèles économiques, choix politiques, Esprit, n 376, pp. 79-81.

PHARABOD A.-S. et al., 2013, « La Mise en chiffres de soi : Une approche compréhensive des mesures personnelles ", Réseaux, n 177, pp. 97-129.

PIOTET D., 2011, « Comment les réseaux sociaux changent-ils notre vie », Esprit (juillet), $\mathrm{n}^{\circ} 376$, pp. 82-95.

PISANI F. et PIOTET D., 2008, Comment le Web change le monde : L'Alchimie des multitudes, Paris, Pearson.

RAMONET I., 2011, « Automates de l'information », Le Monde diplomatique (mars), p. 27. 
REIGELUTH T., 2014, « Why Data is not Enough: Digital Traces as Control of Self and Selfcontrol », Surveillance \& Society, vol. 12, $\mathrm{n}^{\circ}$ 2, pp. 243-354, www.surveillance-and-society.org. RosanVallon P., 2006, La Contre-Démocratie : La Politique à l'âge de la défiance, Paris, Le Seuil. SEN A., 1993, « Capability and Well-Being », in M. NUSSBAUM et A. SEN (éd.), The Quality of Life, Oxford, Clarendon Press, pp. 30-53.

VAIDHYANATHAN S., 2008, « Naked in the Nonopticon », The Chronicle of Higher Education, http:// www.chronicle.com/article/naked-in-the-nonopticon/6197, dernière consultation le 29 février 2016.

WARK M., 2013, « Nouvelles Stratégies de la classe vectorialiste », Multitudes, n 54, pp. 191-198.

\section{NOTES}

1. Pour Dardot et Laval (2009) le gouvernement des sujets (représentations, ressentiments, actions, conduites...) mis en œuvre par les stratégies de pouvoir dominantes s'apparente, de plus en plus, à l'intériorisation massive des normes de l'entreprise (efficacité, compétitivité, performance, production de jouissance) au niveau individuel, à tel point que les acteurs se représentent la poursuite de leur propre désir sous le règne de l'entreprise d'eux-mêmes dans l'«ultrasubjectivité » du "plus-dejouir ». Chacun doit ainsi évaluer son « capital humain », au service duquel il déploie des stratégies promotionnelles, communicationnelles, flexibles en vue d'atteindre une rentabilité, une jouissance et une performance en conformité avec les valeurs dominantes marchandes de la reconnaissance sociale actuelle.

2. Le parcellitarisme est la forme des sociétés occidentales actuelles qui, à la manière d'un totalitarisme à l'envers, «tend à transformer toute chose, tout être ou toute pensée en parcelles soumises à la loi du mouvement brownien des particules élémentaires » (Caillé, 2006, p. 88).

3. Il faut néanmoins relativiser cette interprétation générationnelle. En effet, si on établit des " comparaisons entre enfants de familles aisées et de classes populaires ", on constate "une divergence parfois radicale en termes de compétences informatiques" et d' « habitus informationnel » (Casilli, 2011).

4. «Les enquêtes interrogeant des étudiants sur leur perception des risques pris à dévoiler leur identité sur les réseaux sociaux montrent qu'à leurs yeux la menace réside d'abord dans les figures d'autorité qui leur sont proches, les parents, coachs ou professeurs, bien avant les entreprises et le gouvernement " (Cardon, 2012, p. 24). Ainsi, "décentralisée et distribuée, la surveillance devient un contrôle que chacun ne cesse d'effectuer sur les autres et sur soi-même » (ibid., p. 25).

5. «Si Big Brother était l'incarnation de la société de l'information des années 1980, l'actuel réseautage social hype peut-il être considéré comme l'incarnation du désir latent d'un regard bienveillant et fraternel (de type frère à frère), ou encore comme un acte innocent de voyeurisme amical ?» (Christensen, 2010, p. 54). 
6. «À l'organisation panoptique du pouvoir (par l'enfermement et la discipline), succède une organisation rythmique à finalité mercantile qui repose sur deux rythmes: l'accélération, qui se traduit par une hypermobilisation des corps et des esprits, et la synchronisation autour d'objets industriels techniques capables de capter l'attention des consciences et de les river sur les mêmes contenus culturels alors même qu'ils ont la conviction de s'orienter librement dans le choix des programmes » (Fernandez, 2011, p. 345).

7. Pour certains penseurs critiques (entre une vision habermasienne et foucaldienne), la "vie privée objectivée " par le capitalisme informationnel cherche à s'imposer à la « vie privée subjective » et "vécue " "afin de les rendre compatibles avec les dispositifs de surveillance qui nourrissent le capitalisme informationnel » (Coll, 2014, p. 59). Dès lors, la protection de la vie privée mise en œuvre par les Etats, ONG et grandes entreprises peut elle-même être vue comme un "outil de gouvernance des individus" qui "sert la surveillance plutôt que seulement la limiter ». Car « définir avec le plus d'acuité possible ce qu'est la vie privée reviendrait à la rendre plus facilement saisissable et donc contrôlable ». La vie privée serait dès lors le "partenaire privilégie de la surveillance plutôt que son antidote » (ibid., p. 60).

8. Les technologies DPI («inspection en profondeur des paquets»), par exemple, commercialisés par de grandes entreprises occidentales, sont de gigantesques systèmes de "surveillance des réseaux de communication" (Champagne, 2012). Ces services bénéficient à de multiples acteurs économiques et politiques: aux «opérateurs de télécommunications » ou de téléphonie mobile pour brider certaines connexions trop couteuses ou «allouer une bande passante supérieure à certains services (par exemple, ceux qu'ils éditent...)», pour "garder une trace» de vos préférences afin de commercialiser ces données et/ou créer des " programmes de fidélisation-surveillance »; aux «sociétés d'ayants droit» et aux «détenteurs de copyright» souhaitant «lutter contre les échanges de fichiers illégaux sur les réseaux en pair-à-pair ou les sites de téléchargement direct, du type Megaupload »; ou bien encore aux États autoritaires ou démocratiques « qui souhaitent écouter toute la population » (ibid).

9. Frontières intelligentes et dématérialisées qui n'empêchent pas le retour en force des frontières nationales, physiques et territorialisées classiques, notamment avec la récente « crise des migrants » en Europe. On voit à quel point d'anciens modes de contrôle et de souveraineté coexistent, s'hybrident et se renforcent autant qu'ils ne s'opposent.

10. Avec le Web, «la base de données est devenue une forme sociotechnique omniprésente. Non seulement la Toile donne accès à une myriade de bases, mais elle les met en réseaux. Les moteurs de recherche, qui permettent à chacun de trouver à tout moment l'information dont il a besoin, reposent sur des crawlers qui transforment le Web en une immense base de données » (Flichy et Parasie, 2013, p. 13).

11. M. Castells met notamment en avant l'explosion de la " politique du scandale » liant les réseaux sociaux et les médias télévisuels. «Internet ouvre la communication de masse à des allégations et à des dénonciations issues de sources multiples » (2013, p. 322). « La mise en réseaux des ragots numériques les amplifie dans des proportions gigantesques » ( ibid., p. 322).

12. "La Chine ou la Russie pratiquent aussi la propagande sur Internet », en formant des blogueurs à cet effet, et "qui peut parfois être plus efficace encore que la censure " (Morozov in Millot, 2011). 
13. Outre les actions répressives de la cyberpolice chinoise, « chacun doit devenir un flic à son niveau, jusqu'à l'internaute qui, innovation de 2007, trouve sur les forums des principaux sites, une petite icône représentant un sympathique "cyberpolicier" sur lequel on peut cliquer pour signaler un contenu illicite » (Haski, 2008, p. 32).

14. «L'Occident développe la plupart de ces technologies qui permettent aux régimes autoritaires de contrôler leurs dissidents » (Morozov in Millot, 2011).

15. Avec les données numériques récoltées en masse, on "s'intéresse moins aux raisons profondes qui président à la marche du monde qu'aux associations susceptibles de relier entre eux des phénomènes disparates " (Mayer-Schönberger et Cukier, 2013, p. 20). Il s'opère ainsi un "glissement de la causalité vers la corrélation ».

16. Neuf entreprises seraient concernées: AOL, Apple, Facebook, Google (et Youtube), Microsoft (et Skype), Paltank et Yahoo !. Elles ont toutes démenti avoir participé à ce programme ou avoir installé des «portes dérobées » permettant un accès direct par la NSA.

17. Cette surveillance (plus ou moins méconnue) des données personnelles fait l'objet de réactions contradictoires: de nombreuses et retentissantes controverses médiatisées émergent depuis l'affaire Snowden, mais un «haut niveau d'acceptation sociale des technologies de surveillance» existe chez les populations occidentales qui freine l'apparition de mouvements de contestation et d'indignation majeurs (Chardel, 2014).

18. La dataveillance est le contrôle systématique des gens ou des groupes au moyen de systèmes de données personnelles pour régler ou diriger leur comportement, les entreprises notamment rassemblant, traitant, analysant et utilisant des données pour réaliser leurs objectifs d'affaires.

19. «La classe vectorialiste est donc constituée par tous ceux qui contrôlent et qui profitent de la nécessaire vectorialisation matérielle de l'information - que ce soit à travers la production industrielle d'iPads, de câbles ou de microprocesseurs (Foxconn, Sony, Apple), à travers le déploiement de réseaux de communication monopolisés par des multinationales privées (Orange, Verizon, Google, Facebook), à travers la marchandisation de l'information elle-même par les artifices légaux de la propriété intellectuelle (Microsoft, Universal) ou à travers le contrôle des vecteurs par lesquels passe la financiarisation des investissements qui abreuvent toutes ces entreprises (Goldman Sachs) » (Citton, 2013, p. 168).

20. «Le monde actuel considère désormais comme normale la fétichisation non plus d'un produit mais d'une marque dont on veut tout savoir, tout posséder, comme s'il s'agissait d'une star » (Azam, 2013, p. 30).

21. L'« immense gisement de données numériques découle de la capacité à paramétrer des aspects du monde et de la vie humaine qui n'avaient encore jamais été quantifiés » (Mayer-Schönberger et Cukier, 2013, p. 1). «Les lunettes élaborées par Google - équipées d'une caméra, d'un micro et connectées à Internet - changent notre regard en données ; Twitter met en données nos pensées; LinkedIn fait de même avec nos relations professionnelles » (ibid., p. 21). 


\section{ABSTRACTS}

Horizontal panopticism increasingly appears as the typical shape of power in a networked society. This article tries to understand how online reticular panopticism emerges at the intersection of digital social media, cognitive capitalism and digital giants, neoliberal governance and the contemporary democratic dynamic of recognition. It is characterized by the control and monitoring of all by all on networks through the quest for online visibility and celebrity, the renewal of state monitoring systems via the Internet, and the control of consumers by the business world. This article shows how horizontal panopticism is, to some degree, inversely symmetrical to the totalitarian panopticon model, a model that it is simultaneously very distant from yet at the same time and very close to.

Le panoptisme horizontal apparaît de plus en plus clairement comme la forme prise par le pouvoir dans la société en réseaux. Cet article tente de comprendre comment ce panoptisme réticulaire en ligne émerge à l'intersection des médias sociaux numériques, du capitalisme cognitif et des géants du numérique, de la gouvernementalité néolibérale et de la dynamique démocratique contemporaine de la reconnaissance. Il se caractériserait par la surveillance et le contrôle de tous par tous sur les réseaux via la quête de visibilité/célébrité en ligne, le renouveau des dispositifs de surveillance étatiques par le biais d'Internet et le contrôle marchand des consommateurs. Cet article montre que ce panoptisme horizontal est en partie le symétrique inversé du modèle panoptique de type totalitaire, vis-à-vis duquel il est à la fois très éloigné et très proche.

El panoptismo horizontal se muestra cada vez de forma más diáfana como la forma que adquiere el poder en la sociedad de las redes. Este artículo busca explicar cómo este panoptismo reticular en línea surge en la intersección de varios factores: las redes sociales digitales, el capitalismo cognoscitivo y los gigantes digitales, la manera neoliberal de gobernar y la dinámica contemporánea del reconocimiento. Se caracteriza por la vigilancia y el control de todos por todos, en las redes, a través de la búsqueda de visibilidad/fama en línea, de la renovación de los dispositivos de vigilancia estatales vía Internet, y del control mercantil de los consumidores. Este artículo muestra que este panoptismo horizontal es en parte el simétrico invertido del modelo panóptico de tipo totalitario, respecto al cual se sitúa tan cerca como lejos.

\section{INDEX}

Mots-clés: panoptisme, reconnaissance, médias sociaux, surveillances numériques, aliénation

Palabras claves: panoptismo, reconocimiento, medios sociales, vigilancia digital, alienación Keywords: panopticism, recognition, social medias, digital monitoring, alienation 


\section{AUTHOR}

SIMON BOREL

Simon Borel est docteur en sociologie diplômé de l'université Paris Ouest Nanterre La Défense. Spécialiste des discours et de l'idéologie des réseaux, il travaille sur l'émergence d'une socialité virtuelle-monde (nouveaux rapports sociaux et formes d'individuation en ligne) du point de vue des problématiques de la reconnaissance, du don, de l'idéal et de la dynamique démocratiques et des nouvelles formes économiques contributives et collaboratives propres au capitalisme cognitif contemporain. Il a fait paraître en 2014 un livre, Et les réseaux sauveront le monde... ?, synthétisant ses travaux de recherche. 